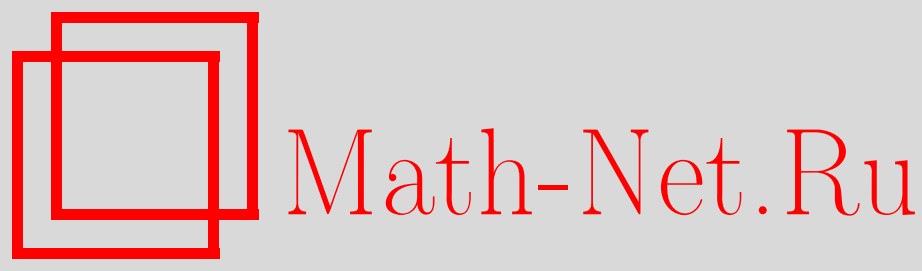

Н. М. Хатамов, Г. Т. Мадгозиев, Меры Гиббса для обобщенной модели Поттса с радиусом взаимодействия два на дереве Кэли, ТМФ, 2015, том 183, номер 3, 450-459

DOI: https://doi.org/10.4213/tmf8702

Использование Общероссийского математического портала Math-Net.Ru подразумевает, что вы прочитали и согласны с пользовательским соглашением http://www.mathnet.ru/rus/agreement

Параметры загрузки:

IP : 54.198 .187 .58

26 апреля 2023 г., 18:00:40

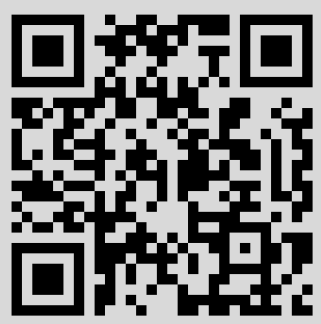




\title{
МЕРЫ ГИББСА ДЛЯ ОБОБЩЕННОЙ МОДЕЛИ ПОТТСА С РАДИУСОМ ВЗАИМОДЕЙСТВИЯ ДВА НА ДЕРЕВЕ КЭЛИ
}

\begin{abstract}
Изучается обобщенная модель Поттса на дереве Кэли порядка $k=3$. При некоторых условиях на параметры показано, что существует не более двух трансляционно-инвариантных мер Гиббса и континуум мер Гиббса, не являющихся трансляционно-инвариантными. Для любого нормального делителя $\widehat{G}$ индекса два группового представления дерева Кэли изучены $\widehat{G}$-периодические меры Гиббса. Доказано, что существует несчетное множество $\widehat{G}$-периодических (не трансляционно-инвариантных и не "шахматно"-периодических) мер Гиббса.
\end{abstract}

Ключевые слова: дерево Кэли, конфигурация, обобщенная модель Поттса, мера Гиббса.

DOI: $10.4213 / \operatorname{tmf} 8702$

\section{1. ВВЕДЕНИЕ}

Одна из основных проблем статистической физики - найти для данного гамильтониана все отвечающие ему меры Гиббса. Определение меры Гиббса и других связанных с ней понятий можно найти, например, в монографиях [1]-[4]. Напомним, что обобщенная модель Поттса является новой и поэтому изучена очень мало по сравнению с другими моделями. Например, модель Поттса хорошо изучена на решетке $\mathbb{Z}^{d}$ и на дереве Кэли.

В работе [5] рассматривалась ферромагнитная модель Поттса с тремя состояниями на дереве Кэли второго порядка и показано, что существует критическая

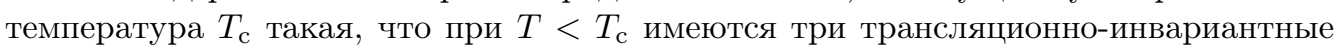
меры Гиббса и несчетное число мер Гиббса, не являющихся трансляционно-инвариантными. В работе [6] результаты статьи [5] обобщены на случай модели Поттса с конечным числом состояний на дереве Кэли произвольного (конечного) порядка. В работе [7] доказано, что на дереве Кэли трансляционно-инвариантная мера Гиббса антиферромагнитной модели Поттса с внешним полем единственна. Работа [8] посвящена модели Поттса со счетным числом состояний и с ненулевым внешним полем на дереве Кэли. Доказано, что эта модель имеет единственную трансляционно-

${ }^{*}$ Наманганский государственный университет, Наманган, Узбекистан.

E-mail: nxatamov@mail.ru 
инвариантную меру Гиббса. В работе [9] найдены все трансляционно-инвариантные меры Гиббса и, в частности, показано, что при достаточно низких температурах их количество равно $2^{q}-1$ (где $q \geqslant 2$ есть число значений спина). Доказано, что существуют $[q / 2]$ критических температур и найдено точное количество трансляционноинвариантных мер Гиббса для каждой промежуточной температуры. В работе [10] изучена проблема крайности трансляционно-инвариантных мер Гиббса, описанных в работе [9]. В работе [11] вводится слабо периодическая мера Гиббса и для модели Изинга найдены некоторые такие меры, а в работе [12] для модели Поттса изучены слабо периодические основные состояния и слабо периодические меры Гиббса. Полученные в работе [12] слабо периодические меры Гиббса также являются трансляционно-инвариантными.

Обобщенная модель Поттса на дереве Кэли рассмотрена в работах [13], [14]. В работе [13] для этой модели на дереве Кэли второго порядка показано, что найдется значение $\lambda_{\text {cr }}$ такое, что при $\lambda>\lambda_{\text {cr }}$ существует не менее трех трансляционно-инвариантных мер Гиббса и несчетное множество $\widehat{G}$-периодических (не трансляционноинвариантных и не "шахматно"-периодических) мер Гиббса.

В настоящей работе обобщены результаты работы [13] на случай дерева Кэли третьего порядка. Содержание работы состоит в следующем: во введении даются основные определения; в разделе 2 приведена система функциональных уравнений; в разделе 3 для рассматриваемой модели доказано существование трансляционноинвариантных мер Гиббса; в разделе 4 изучены периодические меры Гиббса, основной результат этого раздела составляет теорема 4, утверждение которой отражает новое явление в рамках рассматриваемой модели, так как для ранее изученных моделей не существовало $H_{A}$-периодических мер Гиббса при $H_{A} \neq G_{k}^{(2)}$.

Основные понятия. Дерево Кэли $\Gamma^{k}=(V, L)$ порядка $k \geqslant 1$ есть бесконечное дерево, т. е. граф без циклов, из каждой вершины которого выходит ровно $k+1$ ребер. Здесь $V$ - множество вершин дерева $\Gamma^{k}, L-$ множество его ребер. Пусть $i$ - функция инцидентности, сопоставляющая каждому ребру $l \in L$ его концевые точки $x, y \in V$. Если $i(l)=\{x, y\}$, то вершины $x$ и $y$ называются ближайшими соседями, что обозначается как $\langle x, y\rangle$. Расстояние $d(x, y), x, y \in V$, на дереве Кэли определяется формулой

$$
d(x, y)=\min \left\{d \mid \exists x=x_{0}, x_{1}, \ldots, x_{d-1}, x_{d}=y \in V\right\},
$$

где $\left\langle x_{0}, x_{1}\right\rangle, \ldots,\left\langle x_{d-1}, x_{d}\right\rangle$, т.е. $x=x_{0}, x_{1}, \ldots, x_{d-1}, x_{d}=y$ являются ближайшими соседями. Как уже отмечалось, мы изучаем случай $k=3$.

Известно [4], [15], что дерево Кэли представляется как группа $G_{k}$, являющаяся свободным произведением $k+1$ циклических групп второго порядка с образующими $a_{1}, a_{2}, \ldots, a_{k+1}$.

Рассмотрим модель, в которой спин принимает значения из множества $\Phi=\{-1,1\}$. Тогда конфигурация $\sigma$ на $V$ определяется как функция $x \in V \rightarrow \sigma(x) \in \Phi$; множество всех конфигураций совпадает с $\Omega=\Phi^{V}$. Пусть $A \subset V$. Обозначим через $\Omega_{A}$ пространство конфигураций, определенных на множестве $A$. Через $|A|$ обозначим число элементов множества $A$.

Обобщенный символ Кронекера как функция

$$
U\left(\sigma_{A}\right): \Omega_{A} \rightarrow\{|A|-1,|A|-2, \ldots,|A|-\min (|A|, \mid \Phi)\}
$$


определяется следующим образом:

$$
U\left(\sigma_{A}\right)=|A|-\left|\sigma_{A} \cap \Phi\right|,
$$

где $A \subset V$, a $\left|\sigma_{A} \cap \Phi\right|$ - число различных значений $\sigma_{A}(x), x \in A$ (см. работу [14]). В настоящей работе мы рассмотрим случай $|A|=5$.

Обозначим через $M$ множество всех шаров $b(x)=\{y \in V \mid d(x, y) \leqslant 1\}$ с радиусом 1. Гамильтониан модели определяется следующим образом:

$$
H(\sigma)=-J \sum_{b \in M} U\left(\sigma_{b}\right), \quad J \in \mathbb{R} .
$$

\section{2. СИСТЕМА ФУНКЦИОНАЛЬНЫХ УРАВНЕНИЙ}

Пусть $x^{0} \in V$ есть фиксированная точка. Будем писать $x<y$, если путь из $x^{0}$ в $y$ проходит через $x$. Точка $y$ называется прямым потомком точки $x$, если $x<y$ и $d(x, y)=1$. Для $x \in G_{k}$ введем обозначение $x_{\downarrow}=\left\{y \in G_{k} \mid\langle x, y\rangle\right\} \backslash S(x)$, где $S(x)-$ множество прямых потомков точки $x \in V$.

Положим

$$
W_{n}=\left\{x \in V \mid d\left(x^{0}, x\right)=n\right\}, \quad V_{n}=\left\{x \in V \mid d\left(x^{0}, x\right) \leqslant n\right\} .
$$

Пусть (напомним, мы изучаем дерево Кэли порядка $k=3$ )

$$
b(x)=\left\{x, x a_{1}, x a_{2}, x a_{3}, x a_{4}\right\}, \quad \sigma_{b(x)}=\left\{\sigma(x), \sigma\left(x a_{1}\right), \sigma\left(x a_{2}\right), \sigma\left(x a_{3}\right), \sigma\left(x a_{4}\right)\right\} .
$$

Рассмотрим вероятностное распределение $\mu^{(n)}$ на множестве $\Omega_{V_{n}}$ :

$$
\mu^{(n)}\left(\sigma_{n}\right)=Z_{n}^{-1} \exp \left\{-\beta H\left(\sigma_{n}\right)+\sum_{x \in W_{n}} h_{b(x), \sigma_{b(x)}}^{\left.\sigma_{b(x \downarrow}\right)}\right\}, \quad \sigma_{n} \in \Omega_{V_{n}},
$$

где

$$
Z_{n}=\sum_{\bar{\sigma}_{n} \in \Omega_{V_{n}}} \exp \left\{-\beta H\left(\bar{\sigma}_{n}\right)+\sum_{x \in W_{n}} h_{b(x), \bar{\sigma}_{b(x)}}^{\bar{\sigma}_{b(x \downarrow)}}\right\}, \quad h_{b, \bar{\sigma}_{b}}^{\bar{\sigma}_{b}} \in \mathbb{R} .
$$

Говорят, что вероятностное распределение $\mu^{(n)}$ (при $n \geqslant 1$ ) согласованно, если

$$
\sum_{\sigma^{(n)}} \mu^{(n)}\left(\sigma_{n-1}, \sigma^{(n)}\right)=\mu^{(n-1)}\left(\sigma_{n-1}\right)
$$

для всех $n \geqslant 1$ и $\sigma_{n-1} \in \Omega_{V_{n-1}}$. В этом случае существует единственная мера $\mu$ на $\Omega_{V}$ такая, что $\mu\left(\left.\sigma\right|_{V_{n}}\right)=\mu\left(\sigma_{n}\right)$, если $\left.\sigma\right|_{V_{n}}=\sigma_{n}$, для всех $n \geqslant 1$ и $\sigma_{n} \in \Omega_{V_{n}}$.

Рассмотрим следующие конфигурации на единичном шаре:

$$
\begin{aligned}
\sigma_{0} & =\{+,+,+,+,+\}, & \sigma_{1} & =\{+,-,+,+,+\}, \quad \sigma_{2}=\{+,+,+,-,-\}, \\
\sigma_{3} & =\{+,+,-,-,-\}, & \sigma_{4} & =\{+,-,-,-,-\}, \\
-\sigma_{0} & =\{-,-,-,-,-\}, & -\sigma_{1} & =\{-,-,-,-,+\}, \quad-\sigma_{2}=\{-,-,-,+,+\}, \\
-\sigma_{3} & =\{-,-,+,+,+\}, & -\sigma_{4} & =\{-,+,+,+,+\} .
\end{aligned}
$$


Положим

$$
\begin{aligned}
& h_{b, \sigma_{0}}^{\sigma_{0}}=h_{b, 0}, \quad h_{b, \sigma_{1}}^{\sigma_{0}}=h_{b, 1}, \quad h_{b, \sigma_{2}}^{\sigma_{0}}=h_{b, 2}, \quad h_{b,-\sigma_{1}}^{\sigma_{1}}=h_{b, 3}, \\
& h_{b,-\sigma_{2}}^{\sigma_{1}}=h_{b, 4}, \quad h_{b,-\sigma_{3}}^{\sigma_{1}}=h_{b, 5}, \quad h_{b, \sigma_{1}}^{-\sigma_{1}}=h_{b, 6}, \quad h_{b, \sigma_{2}}^{-\sigma_{1}}=h_{b, 7}, \\
& h_{b, \sigma_{3}}^{-\sigma_{1}}=h_{b, 8}, \quad h_{b,-\sigma_{0}}^{-\sigma_{0}}=h_{b, 9}, \quad h_{b,-\sigma_{1}}^{-\sigma_{0}}=h_{b, 10}, \quad h_{b,-\sigma_{2}}^{-\sigma_{0}}=h_{b, 11}, \\
& h_{b, \sigma_{0}}^{-\sigma_{0}}=h_{b, 12}, \quad h_{b,-\sigma_{1}}^{-\sigma_{0}}=h_{b, 13}, \quad h_{b,-\sigma_{2}}^{-\sigma_{0}}=h_{b, 14}, \quad h_{b,-\sigma_{3}}^{-\sigma_{0}}=h_{b, 15} .
\end{aligned}
$$

Для $a \in M$ через $b, c, d$ обозначим прямые потомки шара $a$ [15].

Следующая теорема доказывается аналогично теореме 1 из работы [13]. Эта теорема дает необходимые и достаточные условия на $h_{b, i}$, при которых выполняется условие $(2.2)$.

Теорема 1. Пусть $k=3$. Введем обозначения

$$
\beta=\frac{1}{T}, \quad \lambda=e^{J \beta}, \quad y_{a, i}=e^{h_{a, i}-h_{a, 11}}, \quad i=\overline{0,10} .
$$

Вероятностное распределение $\mu^{(n)}\left(\sigma_{n}\right), n=1,2, \ldots$, в (2.1) согласованно тогда и только тогда, когда для любого а $\in$ и имеют место следующие равенства:

$$
\begin{aligned}
& y_{a, 0}=y_{a, 8}= \\
& =\frac{\lambda y_{b, 0}+y_{b, 1}+y_{b, 2}+y_{b, 3}}{y_{b, 0}+y_{b, 1}+y_{b, 2}+y_{b, 3}} \frac{\lambda y_{c, 0}+y_{c, 1}+y_{c, 2}+y_{c, 3}}{y_{c, 0}+y_{c, 1}+y_{c, 2}+y_{c, 3}} \frac{\lambda y_{d, 0}+y_{d, 1}+y_{d, 2}+y_{d, 3}}{y_{d, 0}+y_{d, 1}+y_{d, 2}+y_{d, 3}}, \\
& y_{a, 1}=y_{a, 9}= \\
& =\frac{\lambda y_{b, 0}+y_{b, 1}+y_{b, 2}+y_{b, 3}}{y_{b, 0}+y_{b, 1}+y_{b, 2}+y_{b, 3}} \frac{\lambda y_{c, 0}+y_{c, 1}+y_{c, 2}+y_{c, 3}}{y_{c, 0}+y_{c, 1}+y_{c, 2}+y_{c, 3}} \frac{y_{d, 4}+y_{d, 5}+y_{d, 6}+1}{y_{d, 0}+y_{d, 1}+y_{d, 2}+y_{d, 3}}, \\
& y_{a, 2}=y_{a, 10}= \\
& =\frac{\lambda y_{b, 0}+y_{b, 1}+y_{b, 2}+y_{b, 3}}{y_{b, 0}+y_{b, 1}+y_{b, 2}+y_{b, 3}} \frac{y_{c, 4}+y_{c, 5}+y_{c, 6}+1}{y_{c, 0}+y_{c, 1}+y_{c, 2}+y_{c, 3}} \frac{y_{d, 4}+y_{d, 5}+y_{d, 6}+1}{y_{d, 0}+y_{d, 1}+y_{d, 2}+y_{d, 3}}, \\
& y_{a, 3}=y_{a, 11}= \\
& =\frac{y_{b, 4}+y_{b, 5}+y_{b, 6}+1}{y_{b, 0}+y_{b, 1}+y_{b, 2}+y_{b, 3}} \frac{y_{c, 4}+y_{c, 5}+y_{c, 6}+1}{y_{c, 0}+y_{c, 1}+y_{c, 2}+y_{c, 3}} \frac{y_{d, 4}+y_{d, 5}+y_{d, 6}+1}{y_{d, 0}+y_{d, 1}+y_{d, 2}+y_{d, 3}}, \\
& y_{a, 4}=y_{a, 12}= \\
& =\frac{\lambda y_{b, 4}+y_{b, 5}+y_{b, 6}+1}{y_{b, 0}+y_{b, 1}+y_{b, 2}+y_{b, 3}} \frac{\lambda y_{c, 4}+y_{c, 5}+y_{c, 6}+1}{y_{c, 0}+y_{c, 1}+y_{c, 2}+y_{c, 3}} \frac{\lambda y_{d, 4}+y_{d, 5}+y_{d, 6}+1}{y_{d, 0}+y_{d, 1}+y_{d, 2}+y_{d, 3}}, \\
& y_{a, 5}=y_{a, 13}=\frac{\lambda y_{c, 4}+y_{c, 5}+y_{c, 6}+1}{y_{c, 0}+y_{c, 1}+y_{c, 2}+y_{c, 3}} \frac{\lambda y_{d, 4}+y_{d, 5}+y_{d, 6}+1}{y_{d, 0}+y_{d, 1}+y_{d, 2}+y_{d, 3}}, \\
& y_{a, 6}=y_{a, 14}=\frac{\lambda y_{d, 4}+y_{d, 5}+y_{d, 6}+1}{y_{d, 0}+y_{d, 1}+y_{d, 2}+y_{d, 3}}, \\
& y_{a, 7}=1 .
\end{aligned}
$$

\section{3. ТРАНСЛЯЦИОННО-ИНВАРИАНТНЫЕ МЕРЫ ГИББСА}

В этом разделе мы изучаем трансляционно-инвариантные меры для модели (1.2) на дереве Кэли. Заметим, что трансляционно-инвариантные меры Гиббса соответствуют решениям (2.4) с $y_{a, i}=y_{i}, i=\overline{0,6}$, при всех $a \in M$. В (2.4) положим 
$y_{a, i}=y_{i} \in \mathbb{R}_{+}$для любого $a \in M$ и получим систему уравнений

$$
\begin{aligned}
& y_{0}=\left(\frac{\lambda y_{0}+y_{1}+y_{2}+y_{3}}{y_{0}+y_{1}+y_{2}+y_{3}}\right)^{3}, \\
& y_{1}=\left(\frac{\lambda y_{0}+y_{1}+y_{2}+y_{3}}{y_{0}+y_{1}+y_{2}+y_{3}}\right)^{2} \frac{y_{4}+y_{5}+y_{6}+1}{y_{0}+y_{1}+y_{2}+y_{3}}, \\
& y_{2}=\frac{\lambda y_{0}+y_{1}+y_{2}+y_{3}}{y_{0}+y_{1}+y_{2}+y_{3}}\left(\frac{y_{4}+y_{5}+y_{6}+1}{y_{0}+y_{1}+y_{2}+y_{3}}\right)^{2}, \\
& y_{3}=\left(\frac{y_{4}+y_{5}+y_{6}+1}{y_{0}+y_{1}+y_{2}+y_{3}}\right)^{3}, \quad y_{4}=\left(\frac{\lambda y_{4}+y_{5}+y_{6}+1}{y_{0}+y_{1}+y_{2}+y_{3}}\right)^{3}, \\
& y_{5}=\left(\frac{\lambda y_{4}+y_{5}+y_{6}+1}{y_{0}+y_{1}+y_{2}+y_{3}}\right)^{2}, \quad y_{6}=\frac{\lambda y_{4}+y_{5}+y_{6}+1}{y_{0}+y_{1}+y_{2}+y_{3}},
\end{aligned}
$$

где $\lambda>0$. В общем случае решить систему уравнений (3.1) очень трудно. Поэтому рассмотрим ее в частном случае. Разрешая систему уравнений (3.1) относительно $\lambda$, получаем

$$
\begin{aligned}
& \lambda=\frac{\sqrt[3]{y_{0}}\left(y_{0}+y_{1}+y_{2}+y_{3}\right)-\left(y_{1}+y_{2}+y_{3}\right)}{y_{0}}, \\
& \lambda=\frac{\sqrt{y_{1} / \sqrt[3]{y_{3}}}\left(y_{0}+y_{1}+y_{2}+y_{3}\right)-\left(y_{1}+y_{2}+y_{3}\right)}{y_{0}}, \\
& \lambda=\frac{\left(y_{2} / \sqrt[3]{y_{3}^{2}}\right)\left(y_{0}+y_{1}+y_{2}+y_{3}\right)-\left(y_{1}+y_{2}+y_{3}\right)}{y_{0}}, \\
& \lambda=\frac{\sqrt[3]{y_{4}}\left(y_{0}+y_{1}+y_{2}+y_{3}\right)-\left(y_{5}+y_{6}+1\right)}{y_{4}}, \\
& \lambda=\frac{\sqrt{y_{5}}\left(y_{0}+y_{1}+y_{2}+y_{3}\right)-\left(y_{5}+y_{6}+1\right)}{y_{4}}, \\
& \lambda=\frac{y_{6}\left(y_{0}+y_{1}+y_{2}+y_{3}\right)-\left(y_{5}+y_{6}+1\right)}{y_{4}}, \\
& \sqrt[3]{y_{3}}=\frac{y_{4}+y_{5}+y_{6}+1}{y_{0}+y_{1}+y_{2}+y_{3}} .
\end{aligned}
$$

Приравнивая правые части первых шести уравнений этой системы, имеем

$$
\begin{aligned}
\left(y_{0} \sqrt[3]{y_{0}}-1\right. & \left.+\left(\sqrt{y_{0} y_{2}}+y_{2}+\sqrt{\frac{y_{2}^{3}}{y_{0}}}\right)\left(\sqrt[3]{y_{0}}-1\right)\right) y_{6}^{3}+ \\
& +\left(y_{0}-1\right) y_{6}^{2}+\left(y_{0}-1-y_{0}\left(y_{2}+\sqrt{y_{0} y_{2}}\right)\left(1+\sqrt{\frac{y_{2}}{y_{0}}}\right)\right) y_{6}+ \\
& +y_{0}-1+\sqrt[6]{\frac{y_{2}^{3}}{y_{0}}}\left(y_{0}+\sqrt{y_{0} y_{2}}+y_{2}+\sqrt{\frac{y_{2}^{3}}{y_{0}}}\right)=0 .
\end{aligned}
$$

Это кубическое уравнение относительно $y_{6}$. Хорошо известно [16], что количество положительных корней многочлена не превосходит количества изменений знаков 
его коэффициентов. Используя этот факт для уравнения (3.3), получим следующие утверждения:

1) если

$$
y_{0}>1, \quad \frac{y_{0}-1}{2 y_{0} y_{2}+\sqrt{y_{0} y_{2}}\left(y_{0}+y_{2}\right)}>1
$$

или

$$
0<y_{0}<1, \quad \frac{1-y_{0}}{\sqrt[6]{y_{2}^{3} / y_{0}}\left(y_{0}+\sqrt{y_{0} y_{2}}+y_{2}+\sqrt{y_{2}^{3} / y_{0}}\right)}>1,
$$

то уравнение (3.3) не имеет положительных решений;

2) если

$$
0<y_{0}<1, \quad \frac{1-y_{0}}{\sqrt[6]{y_{2}^{3} / y_{0}}\left(y_{0}+\sqrt{y_{0} y_{2}}+y_{2}+\sqrt{y_{2}^{3} / y_{0}}\right)}<1,
$$

то уравнение (3.3) имеет не более одного положительного решения;

3) если

$$
y_{0}>1, \quad \frac{y_{0}-1}{2 y_{0} y_{2}+\sqrt{y_{0} y_{2}}\left(y_{0}+y_{2}\right)}<1,
$$

то уравнение (3.3) имеет не более двух положительных решений, графический анализ показывает, что в этом случае уравнение (3.1) имеет ровно два положительных решения;

4) если $y_{0}=1$, то уравнение (3.3) имеет единственное решение

$$
y_{6}=\frac{1+y_{2}}{1+\sqrt{y_{2}}} .
$$

Предположим, что для любого $a \in M$

$$
y_{a, 0}=y_{a, 4}, \quad y_{a, 1}=y_{a, 5}, \quad y_{a, 2}=y_{a, 6}, \quad y_{a, 3}=1 .
$$

Тогда из системы (2.4) следует, что

$$
\begin{aligned}
y_{a, 0} & =\frac{\lambda y_{b, 0}+y_{b, 1}+y_{b, 2}+1}{y_{b, 0}+y_{b, 1}+y_{b, 2}+1} \frac{\lambda y_{c, 0}+y_{c, 1}+y_{c, 2}+1}{y_{c, 0}+y_{c, 1}+y_{c, 2}+1} \frac{\lambda y_{d, 0}+y_{d, 1}+y_{d, 2}+1}{y_{d, 0}+y_{d, 1}+y_{d, 2}+1}, \\
y_{a, 1} & =\frac{\lambda y_{b, 0}+y_{b, 1}+y_{b, 2}+1}{y_{b, 0}+y_{b, 1}+y_{b, 2}+1} \frac{\lambda y_{c, 0}+y_{c, 1}+y_{c, 2}+1}{y_{c, 0}+y_{c, 1}+y_{c, 2}+1}, \\
y_{a, 2} & =\frac{\lambda y_{b, 0}+y_{b, 1}+y_{b, 2}+1}{y_{b, 0}+y_{b, 1}+y_{b, 2}+1} .
\end{aligned}
$$

Справедлива следующая

ЛЕмма 1. Имеют место следуюшие утверждения.

1. Пусть $\lambda>1$. Если $y_{a}=\left(y_{a, 0}, y_{a, 1}, y_{a, 2}\right)$ для $a \in M$ является решением системы (3.5), то при любом $a \in M$ для каждого $i=0,1,2$ выполняются неравенства $y_{i}^{-} \leqslant y_{a, i} \leqslant y_{i}^{+}$, где $\left(y_{0}^{-}, y_{0}^{+}, y_{1}^{-}, y_{1}^{+}, y_{2}^{-}, y_{2}^{+}\right)$- решение системы

$$
\begin{array}{ll}
y_{0}^{-}=\left(\frac{\lambda y_{0}^{-}+y_{1}^{-}+y_{2}^{+}+1}{y_{0}^{-}+y_{1}^{-}+y_{2}^{+}+1}\right)^{3}, & y_{0}^{+}=\left(\frac{\lambda y_{0}^{+}+y_{1}^{+}+y_{2}^{-}+1}{y_{0}^{+}+y_{1}^{+}+y_{2}^{-}+1}\right)^{3}, \\
y_{0}^{-}=\left(\frac{\lambda y_{0}^{-}+y_{1}^{-}+y_{2}^{+}+1}{y_{0}^{-}+y_{1}^{-}+y_{2}^{+}+1}\right)^{2}, & y_{0}^{+}=\left(\frac{\lambda y_{0}^{+}+y_{1}^{+}+y_{2}^{-}+1}{y_{0}^{+}+y_{1}^{+}+y_{2}^{-}+1}\right)^{2}, \\
y_{1}^{-}=\frac{\lambda y_{0}^{-}+y_{1}^{-}+y_{2}^{+}+1}{y_{0}^{-}+y_{1}^{-}+y_{2}^{+}+1}, & y_{1}^{+}=\frac{\lambda y_{0}^{+}+y_{1}^{+}+y_{2}^{-}+1}{y_{0}^{+}+y_{1}^{+}+y_{2}^{-}+1} .
\end{array}
$$


2. Пусть $\lambda<1$. Если $y_{a}=\left(y_{a, 0}, y_{a, 1}, y_{a, 2}\right)$ для $a \in M$ является решением системы (3.5), то при любом $a \in M$ для каждого $i=0,1,2$ выполняются неравенства $y_{i}^{-} \leqslant y_{a, i} \leqslant y_{i}^{+}$, где $\left(y_{0}^{-}, y_{0}^{+}, y_{1}^{-}, y_{1}^{+}, y_{2}^{-}, y_{2}^{+}\right)$- решение системь

$$
\begin{array}{ll}
y_{0}^{-}=\left(\frac{\lambda y_{0}^{+}+y_{1}^{+}+y_{2}^{-}+1}{y_{0}^{+}+y_{1}^{+}+y_{2}^{-}+1}\right)^{3}, & y_{0}^{+}=\left(\frac{\lambda y_{0}^{-}+y_{1}^{-}+y_{2}^{+}+1}{y_{0}^{-}+y_{1}^{-}+y_{2}^{+}+1}\right)^{3}, \\
y_{0}^{-}=\left(\frac{\lambda y_{0}^{+}+y_{1}^{+}+y_{2}^{-}+1}{y_{0}^{+}+y_{1}^{+}+y_{2}^{-}+1}\right)^{2}, & y_{0}^{+}=\left(\frac{\lambda y_{0}^{-}+y_{1}^{-}+y_{2}^{+}+1}{y_{0}^{-}+y_{1}^{-}+y_{2}^{+}+1}\right)^{2}, \\
y_{1}^{-}=\frac{\lambda y_{0}^{+}+y_{1}^{+}+y_{2}^{-}+1}{y_{0}^{+}+y_{1}^{+}+y_{2}^{-}+1}, & y_{1}^{+}=\frac{\lambda y_{0}^{-}+y_{1}^{-}+y_{2}^{+}+1}{y_{0}^{-}+y_{1}^{-}+y_{2}^{+}+1} .
\end{array}
$$

Лемма 2. Пусть $y=\left(y_{0}^{-}, y_{0}^{+}, y_{1}^{-}, y_{1}^{+}, y_{2}^{-}, y_{2}^{+}\right)$- решение системъ (3.6). Если $y_{i}^{-}=y_{i}^{+}, y_{j}^{-}=y_{j}^{+} u y_{0}^{-} \neq 1 /(\lambda-1)$, mo $y_{k}^{-}=y_{k}^{+}$nрu $i \neq j \neq k$ для $i, j, k=0,1,2$.

\section{4. ПЕРИОДИЧЕСКИЕ МЕРЫ ГИББСА}

В этом разделе, считая, что выполнено условие (3.4), мы изучаем периодические меры Гиббса. Положим $h_{a, i}=\ln y_{a, i}, i=0,1,2$, и запишем систему (3.5) в следующим виде:

$$
\begin{aligned}
& h_{a, 0}= \ln \left(\frac{\lambda e^{h_{b, 0}}+e^{h_{b, 1}}+e^{h_{b, 2}}+1}{e^{h_{b, 0}}+e^{h_{b, 1}}+e^{h_{b, 2}}+1} \times\right. \\
&\left.\quad \times \frac{\lambda e^{h_{c, 0}}+e^{h_{c, 1}}+e^{h_{c, 2}}+1}{e^{h_{c, 0}}+e^{h_{c, 1}}+e^{h_{c, 2}}+1} \frac{\lambda e^{h_{d, 0}}+e^{h_{d, 1}}+e^{h_{d, 2}}+1}{e^{h_{d, 0}}+e^{h_{d, 1}}+e^{h_{d, 2}}+1}\right), \\
& h_{a, 1}=\ln \frac{\lambda e^{h_{b, 0}}+e^{h_{b, 1}}+e^{h_{b, 2}}+1}{e^{h_{b, 0}}+e^{h_{b, 1}}+e^{h_{b, 2}}+1} \frac{\lambda e^{h_{c, 0}}+e^{h_{c, 1}}+e^{h_{c, 2}}+1}{e^{h_{c, 0}}+e^{h_{c, 1}}+e^{h_{c, 2}}+1}, \\
& h_{a, 2}=\ln \frac{\lambda e^{h_{b, 0}}+e^{h_{b, 1}}+e^{h_{b, 2}}+1}{e^{h_{b, 0}}+e^{h_{b, 1}}+e^{h_{b, 2}}+1} .
\end{aligned}
$$

Мы рассматриваем периодические решения системы (4.1).

ОПРЕДЕЛЕНИЕ 1 . Пусть $H_{0}-$ подгруппа группы $G_{k}$. Совокупность векторов

$$
h=\left\{h_{a}=\left(h_{a, 0}, h_{a, 1}, h_{a, 2}\right), a \in G_{k}\right\}
$$

называется $H_{0}$-периодической, если $h_{y a, i}=h_{a, i}, i=0,1,2$, для всех $a \in G_{k}$ и $y \in H_{0}$.

ОПРЕДЕЛЕНИЕ 2. Мера Гиббса, соответствующая $H_{0}$-периодической совокупности векторов, называется $H_{0}$-периодической.

Определим функцию $F: h=\left(h_{0}, h_{1}, h_{2}\right) \rightarrow F(h) \in \mathbb{R}$ следующим образом:

$$
F(h)=\frac{\lambda e^{h_{0}}+e^{h_{1}}+e^{h_{2}}+1}{e^{h_{0}}+e^{h_{1}}+e^{h_{2}}+1} .
$$

Пусть $G_{k}^{(2)}$ - подгруппа группы $G_{k}$, состоящая из слов четной длины. Очевидно, что $G_{k}^{(2)}$ является подгруппой индекса 2 [4]. Пусть

$$
h_{a, i}=\left\{\begin{array}{lll}
h_{i}, & \text { если } & c_{a} \in G_{2}^{(2)}, \\
h_{i}^{\prime}, & \text { если } & c_{a} \in G_{2} \backslash G_{2}^{(2)},
\end{array} \quad i=0,1,2,\right.
$$


где $c_{a}$ - центр шара $a$. Тогда из (4.1) получаем систему уравнений

$$
\begin{aligned}
h_{0} & =3 \ln \frac{\lambda e^{h_{0}^{\prime}}+e^{h_{1}^{\prime}}+e^{h_{2}^{\prime}}+1}{e^{h_{0}^{\prime}}+e^{h_{1}^{\prime}}+e^{h_{2}^{\prime}}+1}, & h_{0}^{\prime} & =3 \ln \frac{\lambda e^{h_{0}}+e^{h_{1}}+e^{h_{2}}+1}{e^{h_{0}}+e^{h_{1}}+e^{h_{2}}+1}, \\
h_{1} & =2 \ln \frac{\lambda e^{h_{0}^{\prime}}+e^{h_{1}^{\prime}}+e^{h_{2}^{\prime}}+1}{e^{h_{0}^{\prime}}+e^{h_{1}^{\prime}}+e^{h_{2}^{\prime}}+1}, & h_{1}^{\prime} & =2 \ln \frac{\lambda e^{h_{0}}+e^{h_{1}}+e^{h_{2}}+1}{e^{h_{0}}+e^{h_{1}}+e^{h_{2}}+1}, \\
h_{2} & =\ln \frac{\lambda e^{h_{0}^{\prime}}+e^{h_{1}^{\prime}}+e^{h_{2}^{\prime}}+1}{e^{h_{0}^{\prime}}+e^{h_{1}^{\prime}}+e^{h_{2}^{\prime}}+1}, & h_{2}^{\prime} & =\ln \frac{\lambda e^{h_{0}}+e^{h_{1}}+e^{h_{2}}+1}{e^{h_{0}}+e^{h_{1}}+e^{h_{2}}+1} .
\end{aligned}
$$

Теорема 2. Для модели (1.2) при $\lambda>0$ и при условии (3.4) существует единственная $G_{2}^{(2)}$-периодическая мера Гиббса. Более того, эта мера совпадает с транслячионно-инвариантной мерой.

ДоказАтеЛьство. Рассмотрим случай $h_{0}=h_{0}^{\prime}, h_{1}=h_{1}^{\prime}, h_{2}=h_{2}^{\prime}$. Из системы (4.3) имеем

$$
\begin{aligned}
& h_{0}=3 \ln \frac{\lambda e^{h_{0}}+e^{h_{1}}+e^{h_{2}}+1}{e^{h_{0}}+e^{h_{1}}+e^{h_{2}}+1}, \\
& h_{1}=2 \ln \frac{\lambda e^{h_{0}}+e^{h_{1}}+e^{h_{2}}+1}{e^{h_{0}}+e^{h_{1}}+e^{h_{2}}+1}, \\
& h_{2}=\ln \frac{\lambda e^{h_{0}}+e^{h_{1}}+e^{h_{2}}+1}{e^{h_{0}}+e^{h_{1}}+e^{h_{2}}+1} .
\end{aligned}
$$

Эта система уравнений имеет единственное решение $\left(h_{0}^{*}, h_{1}^{*}, h_{2}^{*}\right)$. Действительно, из (4.4) получаем уравнение $e^{4 h_{2}}+(1-\lambda) e^{3 h_{2}}-1=0$. Пусть $e^{h_{2}}=z$, тогда

$$
Q(z)=z^{4}+(1-\lambda) z^{3}-1=0 .
$$

Заметим, что знаки при коэффициентах $1,1-\lambda,-1$ этого уравнения меняются только один раз. Поэтому по вышеупомянутому свойству [16] о числе положительных корней многочлена мы получаем, что уравнение $Q(z)=0$ имеет единственное положительное решение.

Теперь рассмотрим случай $h_{0} \neq h_{0}^{\prime}, h_{1} \neq h_{1}^{\prime}, h_{2} \neq h_{2}^{\prime}$. Из системы (4.3) получаем уравнения

$$
h_{2}=\ln \frac{\lambda e^{3 h_{2}^{\prime}}+e^{2 h_{2}^{\prime}}+e^{h_{2}^{\prime}}+1}{e^{3 h_{2}^{\prime}}+e^{2 h_{2}^{\prime}}+e^{h_{2}^{\prime}}+1}, \quad h_{2}^{\prime}=\ln \frac{\lambda e^{3 h_{2}}+e^{2 h_{2}}+e^{h_{2}}+1}{e^{3 h_{2}}+e^{2 h_{2}}+e^{h_{2}}+1} .
$$

Отсюда следует, что $P(z)=0$, где $z=e^{h_{2}}$, а многочлен $P(z)$ имеет вид

$$
\begin{aligned}
P(z)= & \left(\lambda^{3}+\lambda^{2}+\lambda+1\right) z^{10}-\left(\lambda^{4}-3 \lambda^{2}-3 \lambda-3\right) z^{9}-\left(3 \lambda^{3}-3 \lambda^{2}-6 \lambda-6\right) z^{8}- \\
& -\left(3 \lambda^{3}-9 \lambda-10\right) z^{7}-\left(3 \lambda^{3}+7 \lambda^{2}-7 \lambda-11\right) z^{6}-\left(9 \lambda^{2}-9\right) z^{5}- \\
& -\left(6 \lambda^{2}+6 \lambda-4\right) z^{4}-\left(3 \lambda^{2}+10 \lambda+3\right) z^{3}-(6 \lambda+6) z^{2}-(3 \lambda+5) z-\lambda-3 .
\end{aligned}
$$

Легко получим разложение $P(z)=Q(z) M(z)$, где $Q(z)$ задан выше и

$$
\begin{aligned}
M(z)= & \left(\lambda^{3}+\lambda^{2}+\lambda+1\right) z^{6}+\left(3 \lambda^{2}+3 \lambda+2\right) z^{5}+\left(3 \lambda^{2}+5 \lambda+4\right) z^{4}+ \\
& +\left(2 \lambda^{2}+8 \lambda+4\right) z^{3}+(4 \lambda+8) z^{2}+(3 \lambda+5) z+\lambda+3 .
\end{aligned}
$$

Так как уравнение $Q(z)=0$ имеет единственное решение, а уравнение $M(z)=0$ не имеет решений, уравнение $P(z)=0$ имеет единственное решение. Теорема доказана. 
Число букв $a_{i}, i=\overline{1, k+1}$, участвующих в несократимой записи слова $x$, обозначим через $\omega_{x}\left(a_{i}\right)$. Пусть $\varnothing \neq A \subseteq N_{3}=\{1,2,3,4\}$ и

$$
H_{A}=\left\{x \in G_{2} \mid \sum_{i \in A} \omega_{x}\left(a_{i}\right) \text { - четное число }\right\} .
$$

Очевидно, что $H_{A}$ является подгруппой индекса 2 [4].

Пусть

$$
h_{a, j}=\left\{\begin{array}{lll}
h_{j}, & \text { если } & c_{a} \in H_{A}, \\
h_{j}^{\prime}, & \text { если } & c_{a} \in G_{2} \backslash H_{A},
\end{array} \quad j=0,1,2 .\right.
$$

Заметим, что (см. определение (4.2))

$$
\begin{aligned}
& h_{0}=\left\{\begin{array}{lll}
3 \ln F(h)=2 \ln F(h)+\ln F\left(h^{\prime}\right), & \text { если } & |A|=1, \\
2 \ln F(h)+\ln F\left(h^{\prime}\right)=\ln F(h)+2 \ln F\left(h^{\prime}\right), & \text { если } & |A|=2, \\
3 \ln F\left(h^{\prime}\right)=2 \ln F\left(h^{\prime}\right)+\ln F(h), & \text { если } & |A|=3 ;
\end{array}\right. \\
& h_{0}^{\prime}=\left\{\begin{array}{lll}
3 \ln F\left(h^{\prime}\right)=2 \ln F\left(h^{\prime}\right)+\ln F(h), & \text { если } & |A|=1, \\
2 \ln F\left(h^{\prime}\right)+\ln F(h)=\ln F\left(h^{\prime}\right)+2 \ln F(h), & \text { если } & |A|=2, \\
3 \ln F(h)=2 \ln F(h)+\ln F\left(h^{\prime}\right), & \text { если } & |A|=3 ;
\end{array}\right. \\
& h_{1}=\left\{\begin{array}{lll}
2 \ln F(h)=\ln F(h)+\ln F\left(h^{\prime}\right), & \text { если } & |A|=1, \\
2 \ln F(h)=\ln F(h)+\ln F\left(h^{\prime}\right)=2 \ln F\left(h^{\prime}\right), & \text { если } & |A|=2, \\
2 \ln F\left(h^{\prime}\right)=\ln F\left(h^{\prime}\right)+\ln F(h), & \text { если } & |A|=3 ;
\end{array}\right. \\
& h_{1}^{\prime}=\left\{\begin{array}{lll}
2 \ln F\left(h^{\prime}\right)=\ln F\left(h^{\prime}\right)+\ln F(h), & \text { если } & |A|=1, \\
2 \ln F\left(h^{\prime}\right)=\ln F\left(h^{\prime}\right)+\ln F(h)=2 \ln F(h), & \text { если } & |A|=2, \\
2 \ln F(h)=\ln F(h)+\ln F\left(h^{\prime}\right), & \text { если } & |A|=3 ;
\end{array}\right. \\
& h_{2}=\ln F(h)=\ln F\left(h^{\prime}\right) ; \\
& h_{2}^{\prime}=\ln F\left(h^{\prime}\right)=\ln F(h) .
\end{aligned}
$$

Случай $|A|=4$ соответствует случаю $H_{A}=G_{2}^{(2)}$, изученному выше.

Теорема 3. Для модели (1.2) при условии (3.4) существует несчетное число $H_{A}$-периодических мер Гиббса. Более того, эти меры не являются трансляционноинвариантными и не являются $G_{2}^{(2)}$-периодическими.

ДокАЗАтЕЛьство. Заметим, что система (4.6) имеет решение тогда и только тогда, когда $F(h)=F\left(h^{\prime}\right)$. Отсюда $t_{0}\left(s_{1}-t_{1}\right)+t_{0}\left(s_{2}-t_{2}\right)+\left(t_{1}+t_{2}+1\right)\left(t_{0}-s_{0}\right)=0$, где $t_{i}=e^{h_{i}}, s_{i}=e^{h_{i}^{\prime}}, i=0,1,2$. В этом уравнении положим $s_{1}-t_{1}=\alpha, s_{2}-t_{2}=\gamma$. Тогда получим

$$
s_{1}=t_{1}+\alpha, \quad s_{2}=t_{2}+\gamma, \quad s_{0}=t_{0}+\frac{(\alpha+\gamma) t_{0}}{t_{1}+t_{2}+1}
$$

Из системы (4.6) имеем $t_{1}=t_{2}^{2}, t_{0}=\left(t_{1}+t_{2}+1\right)\left(1-t_{2}\right) /\left(t_{2}-\lambda\right)$ и уравнение $t_{2}^{4}+(1-\lambda) t_{2}^{3}-1=0$. Это уравнение имеет единственное положительное решение $t_{2}^{*}$. 
Отсюда для любых $\alpha, \gamma \in \mathbb{R}$

$$
\begin{gathered}
t_{2}=t_{2}^{*}, \quad t_{1}=t_{1}^{*}=\left(t_{2}^{*}\right)^{2}, \quad t_{0}=t_{0}^{*}=\frac{\left(t_{1}^{*}+t_{2}^{*}+1\right)\left(1-t_{2}^{*}\right)}{t_{2}^{*}-\lambda} \\
s_{1}=s_{1}^{*}(\alpha)=t_{1}^{*}+\alpha, \quad s_{2}=s_{2}^{*}(\gamma)=t_{2}^{*}+\gamma, \quad s_{0}=s_{0}^{*}(\alpha, \gamma)=t_{0}^{*}+\frac{(\alpha+\gamma) t_{0}^{*}}{t_{1}^{*}+t_{2}^{*}+1} .
\end{gathered}
$$

Таким образом, для любых $\alpha, \gamma \in \mathbb{R}$ существует решение системы (4.6)

$$
\left(\ln t_{0}^{*}, \ln t_{1}^{*}, \ln t_{2}^{*}, \ln s_{0}^{*}(\alpha, \gamma), \ln s_{1}^{*}(\alpha), \ln s_{2}^{*}(\gamma)\right) .
$$

Более того, эти решения различны при различных $\alpha$ и $\gamma$. Теорема доказана.

Из полученных выше результатов, применяя методы работ [17], [18], мы без труда можем доказать следующую теорему

Теорема 4. Для модели (1.2) при условии (3.4) существует континуум мер Гиббса, не являющихся транслячионно-инвариантными.

Благодарности. Авторы выражают признательность профессору У. А. Розикову за полезные дискуссии и советы, касающиеся настоящей работы.

\section{Список литературы}

[1] Х.-О. Георги, Гиббсовские меры и фазовые переходь, Мир, М., 1992.

[2] Я. Г. Синай, Теория фазовых переходов. Строгие результаты, Наука, М., 1980.

[3] C. Preston, Gibbs States on Countable Sets, Cambridge Tracts in Mathematics, 68, Cambridge Univ. Press, Cambridge, 1974.

[4] U. A. Rozikov, Gibbs Measures on Cayley Trees, World Sci., Singapore, 2013.

[5] Н. Н. Ганиходжаев, ТМФ, 85:2 (1990), 163-175.

[6] Н. Н. Ганиходжаев, Докл. АН РУз, 1992, № 6-7, 4-7.

[7] Н. Н. Ганиходжаев, У. А. Розиков, ТМФ, 111:1 (1997), 109-117.

[8] N. N. Ganikhodjaev, U. A. Rozikov, Lett. Math. Phys., 75:2 (2006), 99-109.

[9] C. Kuelske, U. A. Rozikov, J. Stat. Phys., 156:1 (2014), 189-200, arXiv: 1310.6220.

[10] C. Kuelske, U. A. Rozikov, Fuzzy transformations and extremality of Gibbs measures for the Potts model on a Cayley tree, arXiv: 1403.5775.

[11] У. А. Розиков, М. М. Рахматуллаев, ТМФ, 160:3 (2009), 507-516.

[12] М. М. Рахматуллаев, ТМФ, 176:3 (2013), 477-493.

[13] У. А. Розиков, Г. Т. Мадгозиев, ТМФ, 167:2 (2011), 311-322.

[14] U. A. Rozikov, J. Stat. Phys., 130:4 (2008), 801-813.

[15] U. A. Rozikov, J. Stat. Phys., 122:2 (2006), 217-235.

[16] V.V. Prasolov, Polynomials, Algorithms and Computation in Mathematics, 11, Springer, Berlin, 2004.

[17] П. М. Блехер, Н. Н. Ганиходжаев, ТВП, 35:2 (1990), 220-230.

[18] У. А. Розиков, ТМФ, 118:1 (1999), 95-104.

Поступила в редакцию 1.05.2014, после доработки 17.09.2014 